\title{
Exosome-derived miR-142-5p remodels lymphatic vessels and induces IDO to promote immune privilege in the tumour microenvironment
}

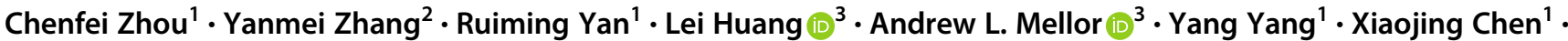

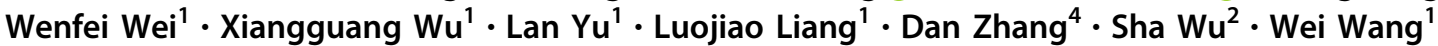

Received: 15 October 2019 / Revised: 31 August 2020 / Accepted: 2 September 2020 / Published online: 14 September 2020

(c) The Author(s) 2020. This article is published with open access

\begin{abstract}
Clinical response to immunotherapy is closely associated with the immunosuppressive tumour microenvironment (TME), and influenced by the dynamic interaction between tumour cells and lymphatic endothelial cells (LECs). Here, we show that high levels of miR-142-5p positively correlate with indoleamine 2,3-dioxygenase (IDO) expression in tumour-associated lymphatic vessels in advanced cervical squamous cell carcinoma (CSCC). The miR-142-5p is transferred by CSCC-secreted exosomes into LECs to exhaust $\mathrm{CD}^{+} \mathrm{T}$ cells via the up-regulation of lymphatic IDO expression, which was abrogated by an IDO inhibitor. Mechanistically, miR-142-5p directly down-regulates lymphatic AT-rich interactive domain-containing protein 2 (ARID2) expression, inhibits DNA methyltransferase 1 (DNMT1) recruitment to interferon (IFN)- $\gamma$ promoter, and enhances IFN- $\gamma$ transcription by suppressing promoter methylation, thereby leading to elevated IDO activity. Furthermore, increased serum exosomal miR-142-5p levels and the consequent IDO activity positively correlate with CSCC progression. In conclusion, exosomes secreted by CSCC cells deliver miR-142-5p to LECs and induce IDO expression via ARID2-DNMT1-IFN- $\gamma$ signalling to suppress and exhaust $\mathrm{CD}^{+} \mathrm{T}$ cells. Our study suggests that LECs act as an integral component of the immune checkpoint(s) in the TME and may serve as a potential new target for CSCC diagnosis and treatment.
\end{abstract}

These authors contributed equally: Chenfei Zhou, Yanmei Zhang, Ruiming Yan

\section{Edited by M. Piacentini}

Supplementary information The online version of this article (https:// doi.org/10.1038/s41418-020-00618-6) contains supplementary material, which is available to authorized users.

$\triangle$ Sha Wu

shawu99@outlook.com

$\triangle$ Wei Wang

smugowwang@126.com

1 Department of Obstetrics and Gynecology, The First Affiliated Hospital of Guangzhou Medical University, Guangzhou 510120, China

2 Department of Immunology/Guangdong Provincial Key Laboratory of Proteomics, School of Basic Medical Sciences, Southern Medical University, Guangzhou 510515, China

3 Institute of Cellular Medicine, Faculty of Medical Sciences, Framlington Place, Newcastle University, Newcastle-UponTyne NE2 4HH, UK

4 Huiqiao Medical Center, Nanfang Hospital, Southern Medical University, Guangzhou 510515, China

\section{Introduction}

Tumour progression has been considered as a consequence of the evolving crosstalk between different cell types within the tumour and its stroma [1-3]. The remodelling of regional lymphatic vessels (LVs) conferred by tumour cells is associated with enhanced malignant progression and poor outcome in many solid tumours, including cervical squamous cell carcinoma (CSCC) [2]. Besides offering physical routes for metastatic spread [4, 5], tumour-associated LVs are thought to be involved in the modulation of adaptive immune responses [6]. These findings have focused research attention on tumour-associated lymphatic endothelial cells (LECs), one of many types of stromal cells, that help establish an immunosuppressive tumour microenvironment (TME), which promotes the evasion of anti-tumour immunity. A better understanding of the immunosuppressive mechanisms of tumour-associated LECs would thus facilitate the rational development of therapeutic strategies.

The recent discovery of miRNAs and their extracellular presence have highlighted the potential role of these regulatory molecules in mediating the cancer-stroma crosstalk [7]. In the 
extracellular space, miRNAs either bind to proteins or lipids [8] or serve as a major RNA component of exosomes [9]. Exosomes are small $30-150 \mathrm{~nm}$ membrane vesicles that are delivered into the extracellular environment by different cell types, including cancer cells [1]. These exosomes reflect the expression patterns of dysregulated miRNAs in cancer cells. Thus, cancer-secreted exosomes may deliver miRNAs to host recipient cells to modify gene expression on a genome-wide scale. The expression of the miR-142-5p that serves as a tumour suppressor has been reported to be down-regulated in gastric cancer [10], while it is up-regulated as a strong oncogene in cutaneous squamous cell carcinoma, colorectal cancer, and renal cell carcinoma [11-13]. However, there are no reports on the cancer-derived exosomal miR-142-5p that may shape the cancer immune landscape and create immune checkpoint(s) in the TME.

Indoleamine 2,3-dioxygenase (IDO) is an intercellular enzyme that converts the essential amino acid tryptophan into kynurenine through the IDO/kynurenine pathway [14]. IDO activity is monitored by measuring kynurenine to tryptophan (K:T) ratio [15]. Elevated IDO activity correlates with a decrease in the population of tumour-infiltrating lymphocytes (TILs) in oesophageal and endometrial cancers $[16,17]$ and poor clinical outcomes in multiple types of cancers [18-20]. These clinical observations support the paradigm that IDO contributes to immune checkpoint development and promotes tumour progression by attenuating effector $\mathrm{T}$ cell responses. Although IDO may be expressed by tumour, stroma, and immune cells in the TME [21, 22], little is known about the molecular mechanism and function of IDO in tumour-associated LVs.

Here, we investigated whether cancer-secreted exosomal miR-142-5p modulates the tumour immune status of CSCC by inducing IDO expression and evaluated the molecular mechanism underlying miR-142-5p-mediated tumour progression to reveal the potential clinical applications of this miRNA in the diagnosis and therapy of CSCC.

\section{Materials and methods}

\section{Cell lines}

The human CSCC cell lines Siha, Caski, C33A, ME180, and MS751 were purchased from the American Type Culture Collection (ATCC) and cultured as per the guidelines. Human dermal lymphatic endothelial cells (HDLECs; IDOnegative) were obtained from ScienCell and cultured in endothelial cell medium (ECM; ScienCell) supplemented with $10 \%$ foetal bovine serum (FBS; Gibco) and endothelial growth medium supplements (ScienCell). In all related experiments, CSCC cell lines and HDLECs were used within 20 and 6 passages, respectively.

\section{Clinical samples}

CSCC tissue microarrays were purchased from SHANGHAI OUTDO BIOTECH CO., LTD. Blood samples were obtained from voluntarily CSCC patients without preoperative radiotherapy or chemotherapy at the department of Gynaecological Oncology of the First Affiliated Hospital of Guangzhou Medical University (Guangzhou, China) or healthy donors between 2016 and 2017. The human study was approved by the World Medical Association Declaration of Helsinki and Institutional Research Ethics Committee at Ministry of Public Health of PR China. Written informed consent was obtained from all patients or healthy donors. All blood samples were centrifuged at $2500 \times \mathrm{g}$ for $10 \mathrm{~min}$ to extract serum. All samples were stored at $-80^{\circ} \mathrm{C}$ until further study.

\section{Exosome isolation, characterisation, and treatment}

Exosomes were purified from CSCC-derived supernatant or the serum of CSCC patients by ultracentrifugation. Cell culture supernatants were collected after $48 \mathrm{~h}$ and centrifuged at $500 \times g$ for $10 \mathrm{~min}$ at $4{ }^{\circ} \mathrm{C}$ and then at $10,000 \times g$ for $30 \mathrm{~min}$ at $4{ }^{\circ} \mathrm{C}$. The serum was diluted with an equal volume of phosphate-buffered saline (PBS) and centrifuged at $2000 \times g$ for $30 \mathrm{~min}$ at $4{ }^{\circ} \mathrm{C}$, followed by $12,000 \times g$ for $45 \mathrm{~min}$ at $4{ }^{\circ} \mathrm{C}$. The supernatants were then passed through $0.22 \mu \mathrm{m}$ filters (Millipore) and ultra-centrifuged at $100,000 \times g$ for $70 \mathrm{~min}$ at $4{ }^{\circ} \mathrm{C}$. The exosome pellets were washed with PBS and subjected to a second ultracentrifugation step at $100,000 \times g$ for $70 \mathrm{~min}$ at $4{ }^{\circ} \mathrm{C}$ and then resuspended in PBS. The exosomes were evaluated by Nanosight NS300 (Malvern). For transmission electron microscopy, exosomes were fixed with $2 \%$ glutaraldehyde and loaded on carbon-coated grids. The grids were then subjected to negative staining using phosphotungstic acid for $2 \mathrm{~min}$ and visualised by transmission electron microscopy (Hitachi H-7500). For the exosome uptake study, PKH67 membrane dye (Sigma) was added to PBS at $1 \mu \mathrm{M}$ concentration and incubated with exosomes for $20 \mathrm{~min}$ before washing. The excess dye was removed by an additional washing step, and the labelled exosomes were resuspended and used to treat HDLECs. After a 48-h incubation, HDLECs were labelled with phalloidin for confocal imaging. For treatment of cells and animals, $2 \mu \mathrm{g}$ exosomes, as measured using a bicinchoninic acid (BCA) protein assay kit (Beyotime), were added to $1 \times 10^{5}$ recipient cells for $48 \mathrm{~h}$.

\section{Real-time quantitative polymerase chain reaction (RT-qPCR)}

The RNA was extracted from cells and exosomes using the miRNeasy Mini Kit (Qiagen) according to the manufacturer's instructions. Reverse transcription was performed using the Mir- $\mathrm{X}^{\mathrm{TM}}$ miRNA First-Strand Synthesis Kit 
(TaKaRa) for miRNAs or PrimeScript ${ }^{\mathrm{TM}} \mathrm{RT}$ Master Mix (TaKaRa) for general genes. RT-qPCR was conducted using SYBR Premix Ex Taq ${ }^{\mathrm{TM}}(\mathrm{TaKaRa})$ on an Applied Biosystems 7500 Fast Real-Time PCR System (Applied Biosystems). Specific primer sets for miR-142-5p and U6 were obtained from RiboBio Inc. The expression of miRNAs and mRNAs was normalised to U6 and GAPDH, respectively. The primer sequences are shown in Supplementary Table S1.

\section{Western blot analysis}

Cell and exosome lysates were prepared in radioimmunoprecipitation assay (RIPA) buffer (FUDE BioTech) and quantified using Bradford protein assay (Beyotime). The lysates were subjected to sodium dodecyl sulphate polyacrylamide gel electrophoresis (SDS-PAGE) and the separated protein bands were transferred onto polyvinylidene fluoride membranes (Millipore). The membranes were incubated with primary antibodies for overnight at $4{ }^{\circ} \mathrm{C}$, and then probed with a specific horseradish peroxidase (HRP)-conjugated anti-rabbit or anti-mouse immunoglobulin-G antibody (ab6721 or ab6728, Abcam). The chemiluminescence signal was detected using the FD8020 FDbio-Dura ECL Kit (FUDE BioTech). The primary antibodies used in this study are shown in Supplementary Table S2.

\section{Fluorescence in situ hybridisation (FISH) and immunohistochemistry (IHC)}

FISH was performed on CSCC tissue microarrays using a fluorescence ISH kit (Boster BioTech) and the miR-142-5p synthetic oligonucleotide probes (Exiqon), according to the manufacturer's protocol. In brief, the slides were incubated with $3 \%$ hydrogen peroxide $\left(\mathrm{H}_{2} \mathrm{O}_{2}\right)$, digested with pepsin for $2 \mathrm{~min}$ at $37^{\circ} \mathrm{C}$, and fixed with $1 \%$ paraformaldehyde (PFA) in diethyl pyrocarbonate (DEPC) for $5 \mathrm{~min}$. The slides were then pre-hybridised in a hybridisation buffer at $42{ }^{\circ} \mathrm{C}$ with miR-142-5p or U6 probes and incubated with fluorescent streptavidin-biotin complex (f-SABC) and horseradish peroxidase polymer. For IHC, sections were immersed in $3 \% \mathrm{H}_{2} \mathrm{O}_{2}$ to block endogenous peroxidase activity and incubated with primary antibodies, overnight at $4{ }^{\circ} \mathrm{C}$. A horseradish peroxidase-conjugated anti-rabbit secondary antibody (ZSGB BioTech) was subsequently applied for $1 \mathrm{~h}$ at room temperature. The expression of D240 (M361929-2, DAKO), IDO (86630, CST), and CD8 (ab93278, Abcam) was visualised using 3,3'-diaminobenzidine (DAB) following counterstaining with haematoxylin (ZSGB BioTech). FISH- and IHC-stained tissue sections were reviewed and scored separately by two independent pathologists. For semi-quantitative evaluation of IDO and miR-142-5p expression in tissue sections, the German Immuno-Reactive Score was applied as previously described [23].

\section{Multiplexed immunofluorescence staining}

Multiplexed immunofluorescence staining was performed by OPAL 4-colour fluorescence IHC Kit (Perkin Elmer) according to manufacturer's protocol. The following primary antibodies were used: D240 (M361929-2, DAKO) and IDO (86630, CST). After deparaffinization, steps were repeated for each primary antibody and sections were microwaved in an antigen retrieval buffer for $45 \mathrm{~s}$ at $100{ }^{\circ} \mathrm{C}$. The slides were washed and blocked for $10 \mathrm{~min}$ at room temperature, and then incubated with a primary antibody. Next, slides were incubated with SuperPicture Polymer Detection Kit-HRP-broad spectrum (Life Technologies), and subsequently treated with Opal fluorochromes (Opal520 and Opal570) diluted 1:150 in an amplification buffer (all provided by the OPAL 4-colour fluorescence IHC kit) for $10 \mathrm{~min}$ at room temperature. Finally, a microwave treatment with AR6 buffer was performed. The slides were then incubated with a $4^{\prime}, 6$ diamidino-2-phenylindole (DAPI) working solution (provided by the OPAL 4-colour fluorescence IHC kit) for $5 \mathrm{~min}$ at room temperature and then mounted under coverslips with ProLong Diamond Antifade Mountant (Life Technologies).

\section{Stable transfection with lentiviral vector}

Lenti-GFP containing an miR-142-5p overexpression sequence and its negative control RNA (miR-NC) were all purchased from GeneChem Inc. Siha and Caski cells were transfected with lenti-GFP/miR-142-5p or lenti-GFP/miRNC. Polyclonal cells with green fluorescent protein signals were purified for further experiments using a fluorescenceactivated cell sorting flow cytometer.

\section{Transient transfection with oligonucleotides and plasmids}

The miR-142-5p mimics and their negative control (miRNC) were designed and cloned by RiboBio Inc. The ARID2 coding sequence (without 3'-UTR) was cloned into the pCDNA3.1(+)-Vector (Invitrogen). The empty vector was used as a blank control. SiARID2 and its negative control (siRNA) were designed and synthesised by GenePharma Inc. Lipofectamine 2000 Reagent (Invitrogen) was then used to transfect miR-142-5p mimics or inhibitors, siARID2, and pCDNA3.1(+)-ARID2 according to the manufacturer's protocol. For RNA extraction, western blot and in vitro assays, cells were used $48 \mathrm{~h}$ after transfection. 
The sequences of siARID2 and siRNA are shown in Supplementary Table S1.

\section{In vivo xenograft model}

Female B-NDG ${ }^{\circledast}$ (NOD- Prkdc $c^{\text {scid }} I L 2 r g^{\text {tml }} / \mathrm{Bcgen}$ ) mice (5weeks-old) were purchased from Beijing Biocytogen Co., Ltd. Siha or Caski cells $\left(1 \times 10^{5}\right)$ mixed with conditioned HDLECs $\left(1 \times 10^{5}\right)$ treated with miR-142-5p mimics or exosomes from Siha/miR-142-5p or Caski/miR-142-5p were randomly injected into the right flank of each mouse ( $n=6$ per group). The tumours were harvested for analysis after 15 days. All animal studies were approved by the Institutional Animal Research Ethics Committee of Guangzhou Medical University.

\section{Human-activated $\mathrm{CDB}^{+} \mathrm{T}$ cell preparation}

Peripheral blood mononuclear cells (PBMCs) were isolated from healthy donors using Ficoll-Paque Plus (GE Healthcare). $\mathrm{CD} 8^{+} \mathrm{T}$ cells were isolated from PBMCs by magnetic bead purification using a human $\mathrm{CD} 8^{+} \mathrm{T}$ cell enrichment kit (STEMCELL Technologies). The purity (> 95\%) was evaluated by flow cytometry using an eF450-labelled antibody against CD8 (48-0087-42, Thermo Fisher). CD8 ${ }^{+}$ $\mathrm{T}$ cells were cultured in complete Roswell Park Memorial Institute (RPMI)-1640 medium. Then, $\mathrm{CD} 8^{+} \mathrm{T}$ cells $(1 \times$ $10^{5}$ ) were activated by stimulation with plate-bound antiCD3 (OKT3, Thermo Fisher) at $2.5 \mu \mathrm{g} / \mathrm{mL}$ and anti-CD28 $(10 \mathrm{~F} 3$, Thermo Fisher) at $2 \mu \mathrm{g} / \mathrm{mL}$ in vitro.

\section{In vitro $\mathrm{CD8}^{+} \mathbf{T}$ cell immune suppression}

HDLECs $\left(1 \times 10^{5}\right)$ treated as indicated with or without $100 \mu \mathrm{M}$ BMS-986205 (HY-101560, MCE) were cocultured with activated $\mathrm{CD} 8^{+} \mathrm{T}$ cells $\left(1 \times 10^{5}\right)$ in the presence of a semipermeable transwell membrane (BD Biosciences). To access the cytotoxic activity of $\mathrm{CD} 8^{+}$ $\mathrm{T}$ cells, the cells from 3-day co-cultures were subjected to surface or intracellular staining using the following antihuman antibodies or controls: fluorescein isothiocyanate (FITC)-conjugated anti-CD69 (11-0699-42, Thermo Fisher), phycoerythrin (PE)-conjugated anti-interferon (IFN)- $\gamma$ (12-7319-42, Thermo Fisher), allophycocyanin (APC)-conjugated anti-programmed cell death protein-1 (PD-1; 17-2799-42, Thermo Fisher), or a fluorochromeconjugated control IgG isotype. For analysis of $\mathrm{CD} 8^{+} \mathrm{T}$ cell apoptosis, the proportion of apoptotic $\mathrm{CD}^{+} \mathrm{T}$ cells was examined using an annexin V-APC apoptosis detection kit (KGF004, KeyGEN Biotech) after 7 days of co-cultures. Flow cytometry was performed using FACS LSRFortessa (BD Biosciences), and data were analysed with FlowJo VX software.

\section{Measurements of tryptophan and kynurenine}

Supernatant or serum levels of tryptophan and kynurenine were measured using high-performance liquid chromatography (HPLC; UltiMate 3000, Thermo Fisher), as previously described with minor modifications [15]. For supernatants, $100 \mu \mathrm{L}$ samples were diluted with a $1 / 10$ volume of $150 \mathrm{mM} \mathrm{NaAc}(\mathrm{pH} 4.0)$ and deproteinated with perchloric acid. For serum samples, $100 \mu \mathrm{L}$ samples were diluted with an equal volume of $30 \mathrm{mM} \mathrm{NaAc}(\mathrm{pH} 4.0)$ and deproteinated with $10 \%$ trichloric acid. In total, $50 \mu \mathrm{L}$ of diluted samples were then loaded and analysed on a C18 column (Thermo Fisher). Tryptophan and kynurenine were detected on a UV channel at 280 and $360 \mathrm{~nm}$, respectively. Samples were analysed using Dionex ${ }^{\mathrm{TM}}$ Chromeleon $^{\mathrm{TM}}$ 7.0 software (Thermo Fisher) and quantified using external standards. At least one quality control sample was randomly inserted into every plate for reference. All assays were performed in triplicates, and each experiment was repeated thrice.

\section{Chromatin immunoprecipitation (ChIP) assay}

Cells were crosslinked with $1 \%$ formaldehyde, quenched in a glycine solution, and subjected to CHIP assay using an enzymatic chromatin IP kit (\#9003, CST) according to the manufacturer's protocol. Anti-AT-rich interactive domain-containing protein 2 (ARID2) (orb386710, Biobyt), anti-DNA methyltransferase 1 (DNMT1) (61467, active motif), and normal IgG (Millipore) antibodies were used for immunoprecipitation. ChIP-enriched DNA samples were quantified by qPCR to determine ARID2- and DNMT1-binding sites in the IFN- $\gamma$ promoter region. Data were shown as relative enrichment following normalisation to control IgG. The primer sequences used for ChIP-qPCR are shown in Supplementary Table S1.

\section{Methylation-specific polymerase chain reaction (MSP)}

MSP was performed according to a previous study [24] using methylated primer pairs designed from the free online tool, MethPrimer (http://www.urogene.org/methprimer/ index 1.html). The methylated (M) and unmethylated (UM) primer sequences are shown in Supplementary Table S1.

\section{Other methods}

Staining assessment, Luciferase activity assay, and ELISA assay were performed using the manufacturer's protocol. See Supplementary Methods for more details. 
A

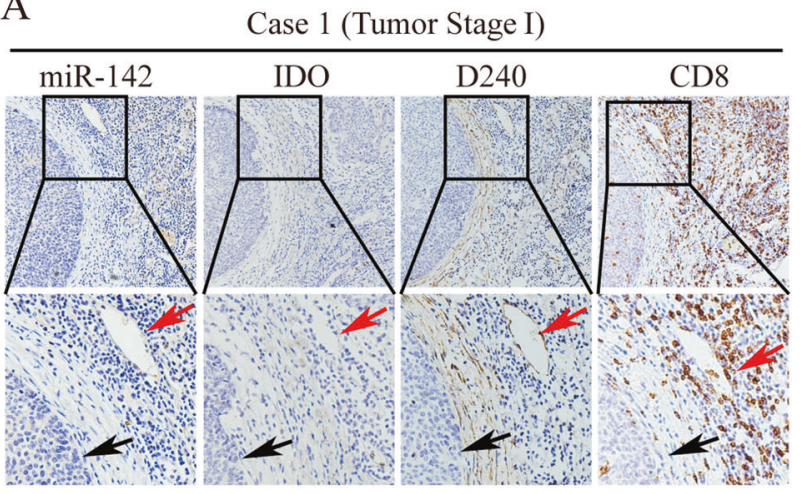

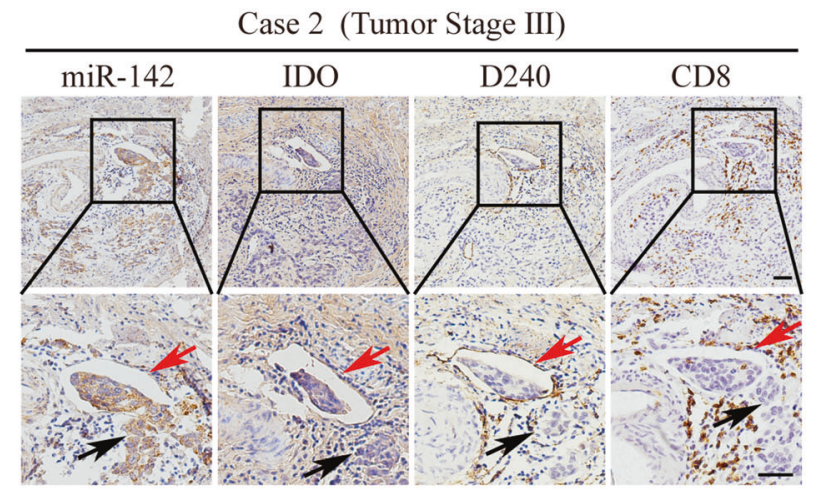

B $\mathrm{C}$

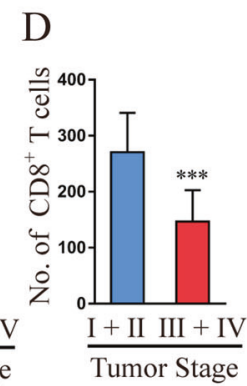

$\mathrm{E}$

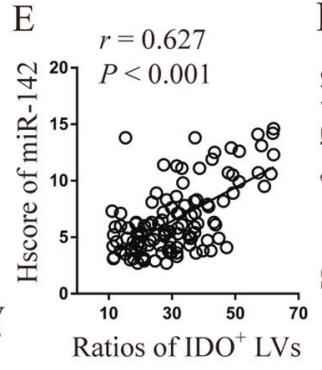

G

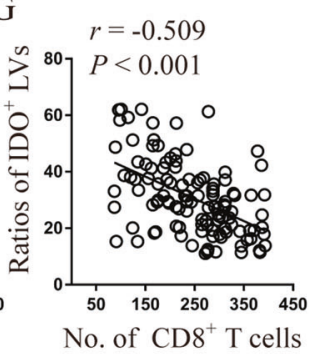

Fig. 1 miR-142-5p expression correlates with lymphatic IDO expression and is negatively associated with the number of infiltrating $\mathrm{CD8}^{+} \mathbf{T}$ cells during $\mathrm{CSCC}$ progression. a Representative micrographs of miR-142-5p, IDO, and D240 (lymphatic marker) staining in serial sections of CSCC specimens are shown. The LVs are indicated by red arrows. The tumour cells are indicated by black arrows. Scale bar: $50 \mu \mathrm{m}$. b-d ISH scores (Hscore) of miR-142-5p,

\section{Statistical analysis}

SPSS V.13.0 software was used for statistical analysis. Quantitative values in all experiments are expressed as the means \pm standard deviation (SD). A two-tailed Student's $t$ test or one-way analysis of variance (ANOVA) was used for comparison between multiple groups. The Fisher or chisquare-test was applied for categorical variables. Correlation analysis was performed using the Spearman rank test. Differences were considered statistically significant at $P<0.05$.

\section{Results}

\section{miR-142-5p expression during CSCC progression correlates with lymphatic IDO expression and is negatively associated with the number of infiltrating $\mathrm{CD8}^{+} \mathrm{T}$ cells}

To evaluate the expression of miR-142-5p during CSCC progression, we analysed 116 human CSCC tissue microarrays using ISH. Compared with those in early stages (FIGO 2018, stages I and II, $n=93$ ), miR-142-5p ratio of $\mathrm{IDO}^{+} \mathrm{LVs}$ to total LVs, and number of $\mathrm{CD} 8^{+} \mathrm{T}$ cells per field were analysed in serial sections from early-stage (FIGO 2018, stages I and II, $n=93$ ) and advanced-stage (FIGO 2018, stages III and IV, $n=$ 23) CSCC patients. e-g Correlation between miR-142-5p level, ratio of $\mathrm{IDO}^{+} \mathrm{LVs}$ to total LVs, and number of $\mathrm{CD}^{+} \mathrm{T}$ cells was analysed. miR-142, miR-142-5p. Error bars represent the mean \pm SD of three independent experiments. $* * * P<0.001$.

expression and the ratios of peritumoral $\mathrm{IDO}^{+}$LVs indicated by both IDO- and D240-positive vessels were significantly higher in advanced stage CSCC patients (FIGO 2018, stages III and IV, $n=23$ ), whereas the number of $\mathrm{CD}^{+} \mathrm{T}$ cells decreased during CSCC progression (Fig. 1a-d). Thus, miR-142-5p expression positively correlated with the ratio of peri-tumoural $\mathrm{IDO}^{+} \mathrm{LVs}$ to total LVs (Fig. 1e). Further, miR-142-5p expression and the ratio of peri-tumoural $\mathrm{IDO}^{+} \mathrm{LVs}$ to total $\mathrm{LVs}$ were negatively associated with the number of tumour-infiltrating $\mathrm{CD} 8^{+}$ $\mathrm{T}$ cells (Fig. 1f, g). As shown in Fig. 1a, high lymphatic miR-142-5p and IDO expression co-localised with high miR-142-5p expression in the tumour, indicating that the tumour-derived miR-142-5p transferred to peri-tumoural LVs may up-regulate lymphatic IDO expression.

\section{miR-142-5p can be transferred to HDLECs via CSCC- secreted exosomes}

Several recent studies have shown that cancer-secreted exosomal miRNAs regulate the crosstalk between malignant and stromal cells in the TME [25]. We, therefore, investigated whether miR-142-5p is involved in the crosstalk between CSCC cells and HDLECs through exosomes. 

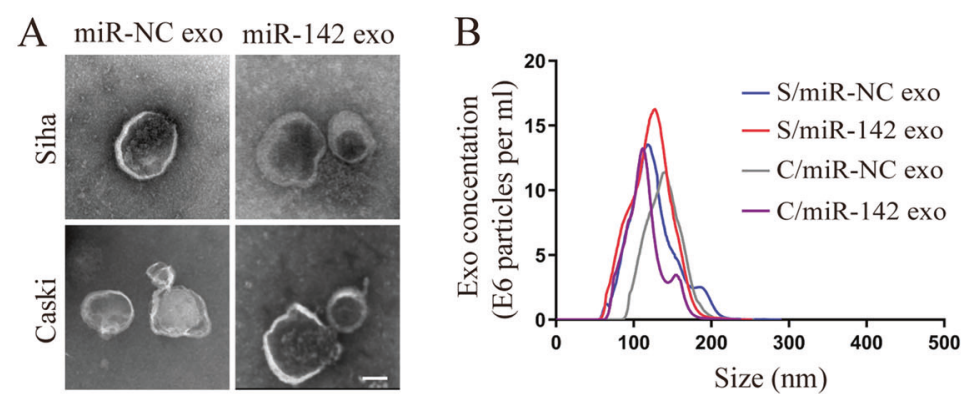

$\mathrm{D}$

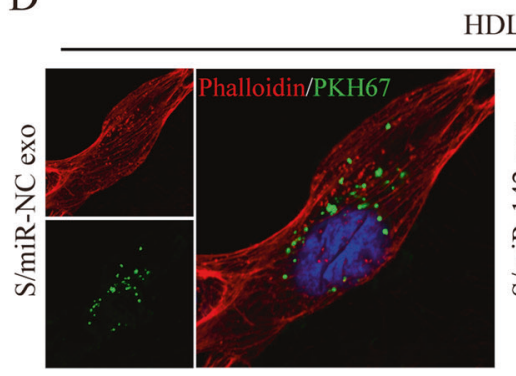

HDLEC
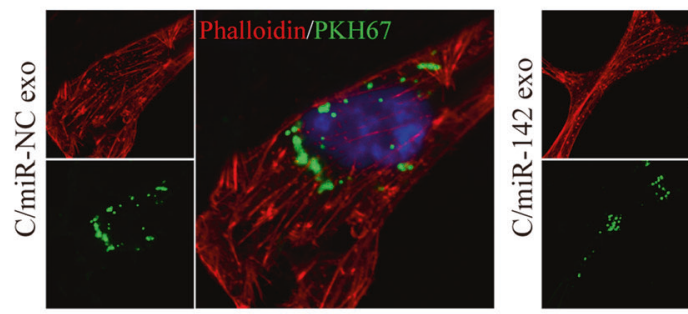
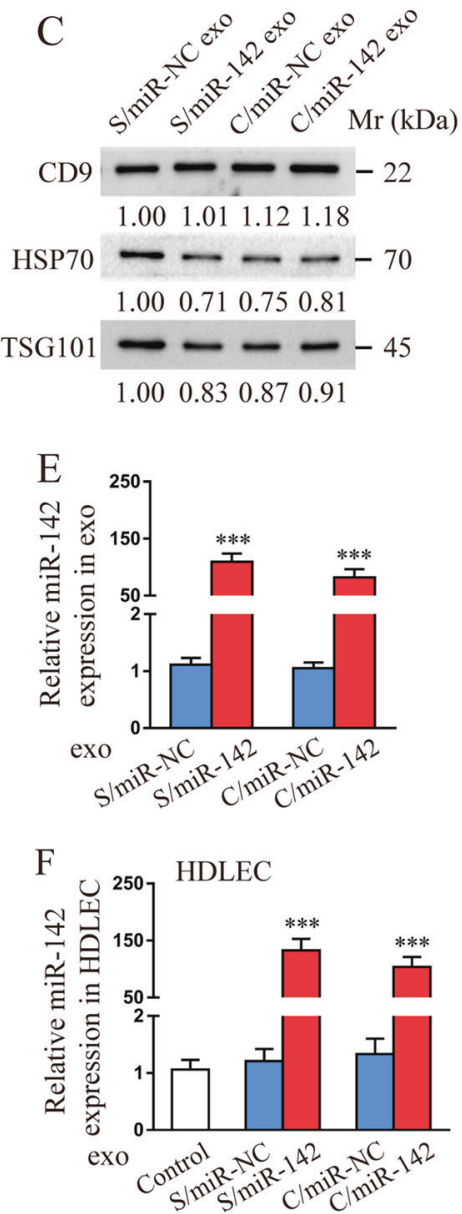

Fig. 2 miR-142-5p can be transferred to human dermal lymphatic endothelial cells (HDLECs) via CSCC-secreted exosomes. a and b The morphology and size of exosomes secreted by Siha and Caski cells transfected with lentivectors overexpressing miR-142-5p or NC were confirmed by transmission electron microscopy and Nanosight particle tracking analysis. Scale bar, $50 \mathrm{~nm}$. c Characteristic marker expression (CD9, HSP70, and TSG101) of exosomes was confirmed by western blotting. d Confocal imaging showed the delivery of
PKH67-labelled exosomes (green) to phalloidin-labelled HDLECs (red). Scale bar, $25 \mu \mathrm{m}$. e miR-142-5p levels in indicated exosomes were detected by RT-qPCR. f miR-142-5p levels in HDLECs pretreated with PBS or indicated exosomes for $24 \mathrm{~h}$ were detected by RTqPCR. S/miR-NC exo Siha/miR-NC exosomes, S/miR-142 exo Siha/ miR-142-5p exosomes, C/miR-NC exo Caski/miR-NC exosomes, C/miR-142 exo Caski/miR-142-5p exosomes. Error bars represent the mean \pm SD of three independent experiments. ${ }^{* * *} P<0.001$.
Evaluation of all CSCC cell lines for of miR-142-5p expression showed that the level of miR-142-5p in Siha and Caski cells was similar to that observed in stage I tumour tissues but significantly lower than that reported in stage III tumour tissues (Supplementary Fig. S1a). Then, a lentiviral vector expressing miR-142-5p or negative control (NC) was successfully constructed and transfected into Siha and Caski cells (Supplementary Fig. S1b). Next, exosomes were isolated and purified from cell culture supernatants using standard ultracentrifugation. The cup-shaped structure, size, and number of isolated exosomes were identified by transmission electron microscopy and Nanosight particle tracking analysis (Fig. 2a, b). The isolated particles were confirmed to be exosomes through the characteristic expression of CD9, heat shock protein 70 (HSP70), and tumour susceptibility 101 (TSG101) (Fig. 2c). To evaluate the delivery of exosomes, the CSCC-secreted exosomes and HDLECs were labelled with PKH67 (green) and phalloidin (red), respectively. After $48 \mathrm{~h}$ incubation, confocal imaging was performed that revealed the appearance of green fluorescent spots in recipient HDLECs, indicating that the labelled exosomes secreted by CSCC cells were delivered to HDLECs (Fig. 2d).

RT-qPCR analysis showed a significant increase in the expression of miR-142-5p in the exosomes secreted by lenti-miR-142-5p-transfected Siha and Caski cells as compared with that in NC (Fig. 2e). To investigate whether the CSCC-secreted miR-142-5p is transferred to HDLECs via exosomes, we detected miR-142-5p level in HDLECs incubated with CSCC-secreted exosomes. The expression of miR-142-5p was higher in HDLECs incubated with exosomes with high miR-142-5p level than in HDLECs 
incubated with exosomes with low miR-142-5p (Fig. 2f), indicating the horizontal transfer of miRNA-142-5p from CSCC cells to HDLECs via exosomes.

\section{CSCC-secreted exosomal miR-142-5p up-regulates lymphatic IDO expression}

To investigate the biological role of exosomal miR-142-5p in mediating lymphatic expression of IDO, we analysed the expression of IDO in HDLECs incubated with CSCC-secreted exosomes for $48 \mathrm{~h}$ by western blotting. IDO expression was significantly up-regulated in HDLECs treated with miR-142$5 p$ mimics or exosomes containing miR-142-5p as compared with that in NC (Fig. 3a). As IDO enzyme degrades tryptophan and generates the tryptophan catabolite kynurenine [15], we assessed the concentrations of tryptophan and kynurenine in culture media using HPLC. An increase in kynurenine to tryptophan (K:T) ratio, indicative of elevated IDO activity, was observed in the supernatant of HDLECs treated with miR$142-5 p$ mimics or exosomes containing miR-142-5p, but not in NC samples (Fig. 3b), consistent with the observation of IDO protein level.

To assess the role of exosomal miR-142-5p in the remodelling of the lymphatic phenotype in vivo, Siha cells mixed with conditioned HDLECs treated with exosomes from Siha/miR-142-5p or miR-142-5p mimics were subcutaneously injected into the right flanks of mice ( $n=6$ per group). After 15 days, the Siha cell group showed higher lymphatic expression of miR-142-5p and IDO (Fig. 3c-e) and serum K:T ratio (Fig. 3f) compared to the control group. Similar results were observed for the group exposed to Caski cells mixed with conditioned HDLECs treated with exosomes from Caski/miR-142-5p or miR-142-5p mimics (Supplementary Fig. S2a-d). In addition, HDLECs mixed with Siha/miR-NC, Siha/miR-142-5p, Caski/miR-NC, or Caski/miR-142-5p were used to construct the above xenograft model. The lymphatic expression of miR-142-5p and IDO (Supplementary Fig. S3a-c) and the serum K:T ratio (Supplementary Fig. S3d) were higher in the group of HDLECs mixed with Siha/miR-142-5p or Caski/miR-142$5 p$ than in the group mixed with Siha/miR-NC or Caski/ miR-NC. Taken together, CSCC-secreted exosomal miR142-5p up-regulates the lymphatic expression of IDO in vitro and in vivo.

\section{CSCC-secreted exosomal miR-142-5p directly targets ARID2 in HDLECs}

Three bioinformatic tools (miRWalk, miRDB, and miRTCDS) were employed to identify the candidate targets of miR142-5p. All three programs predicted CCNT2, RAP1A, ARID2, ITGAV, FIGN, and FAM199X as candidate targets of miR-142-5p. To verify these predictions, we performed RT-
qPCR analyses and found that only ARID2 expression was significantly down-regulated in HDLECs following pretreatment with miR-142-5p mimics (Fig. 4a). We also confirmed the decrease in the protein level of ARID2 in HDLECs treated with miR-142-5p mimics or exosomes containing miR-142-5p (Fig. 4b). We then determined the alignment between miR-142-5p and full-length ARID2 sequences and found an ARID2-coding sequence as a potential target of miR-142-5p (Fig. 4c). The wild-type and mutated miR-142$5 p$-binding sites were cloned into luciferase vectors and the luciferase activity was found to be significantly decreased in HDLECs co-transfected with the vector carrying the wildtype-binding site in the presence of miR-142-5p mimics, but not in HDLECs carrying the mutated-binding site (Fig. 4d). The protein level of ARID2 and luciferase activity also dramatically decreased in HDLECs treated with exosomes containing miR-142-5p (Fig. 4e, f). In addition, ARID2 expression level was examined in 116 human CSCC tissue microarrays using IHC and found to be significantly lower in advanced-stage (FIGO 2018, stages III and IV, $n=23$ ) CSCC patients than in early-stage CSCC patients (FIGO 2018, stages I and II, $n=93$ ) (Supplementary Fig. S4a). Further, ARID2 expression negatively correlated with miR-142-5p level in human CSCC tissues (Supplementary Fig. S4b). Taken together, these results suggest that ARID2 is a direct target of miR-142-5p.

We transfected cells with a plasmid-overexpressing ARID2 without $3^{\prime}$-untranslated region (UTR) or a specific small-interfering RNA (siRNA) SiARID2 that suppressed the expression of ARID2. Western blot analysis confirmed that plasmid-ARID2 and SiARID2 effectively overexpressed and knocked down ARID2 expression in HDLECs, respectively (Fig. 4g). Further in vitro studies confirmed that ARID2 overexpression neutralised the ability of miR-142-5p mimics or exosomes containing miR142-5p to induce IDO activity (Fig. 4g, h). In contrast, ARID2 knockdown replicated the effects of miR-142-5p overexpression in HDLECs by inducing functional IDO expression (Fig. 4g, h). As IFN- $\gamma$ induces IDO expression $[26,27]$, we next assessed whether IFN- $\gamma$ expression was induced by the miR-142-5p-ARID2 axis. miR-142-5p overexpression or ARID2 knockdown significantly upregulated the lymphatic level of IFN- $\gamma$, whereas ARID2 overexpression decreased the up-regulated level of IFN- $\gamma$ induced by miR-142-5p mimics or exosomes containing miR-142-5p (Fig. 4i).

\section{ARID2 recruits DNMT1 to the IFN- $\gamma$ promoter and enhances its methylation}

As ARID2 mediated the expression of IFN- $\gamma$, we explored whether there exists a direct link between ARID2 and IFN- $\gamma$. As shown in Fig. 5a, luciferase reporter assay revealed the 

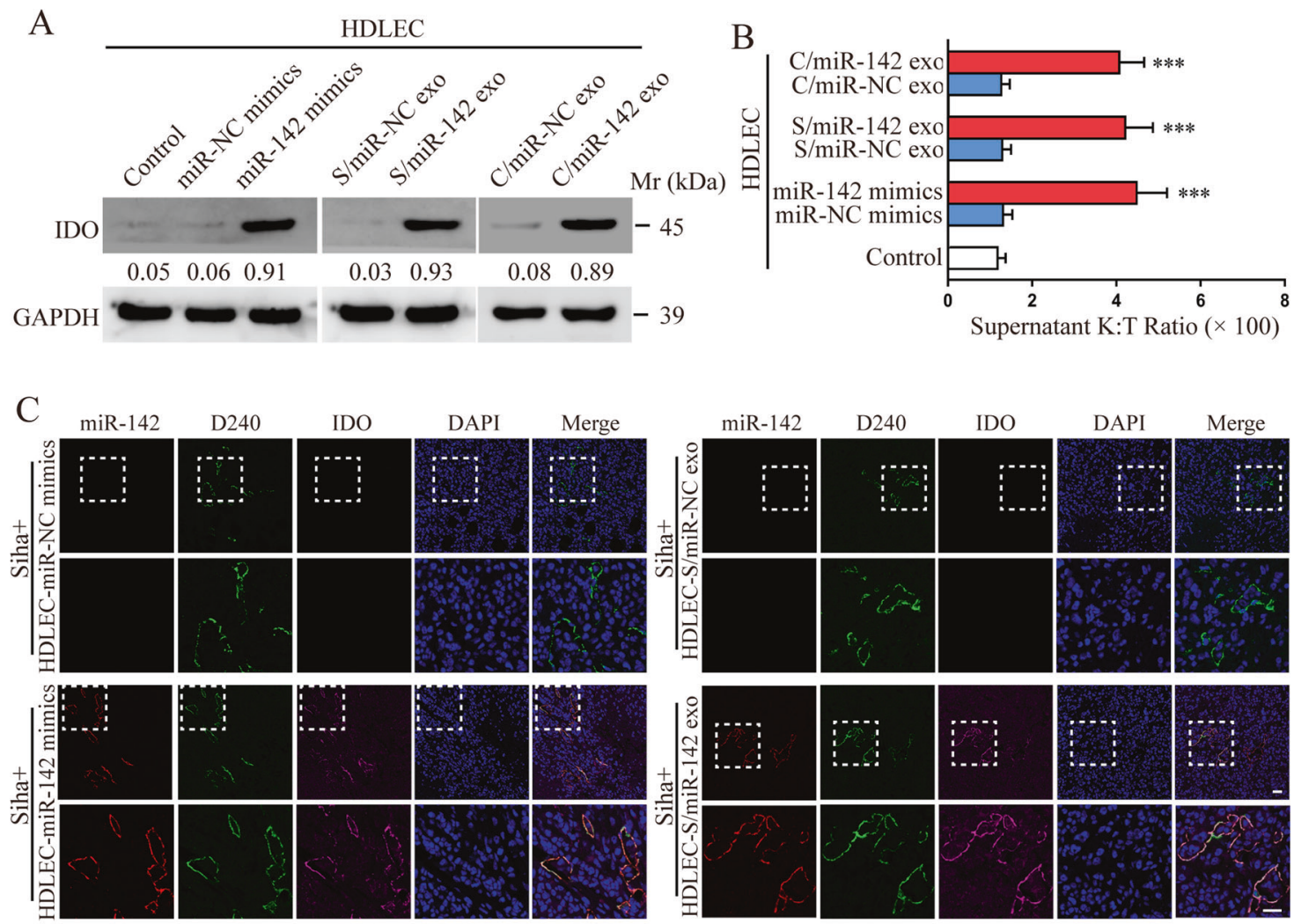
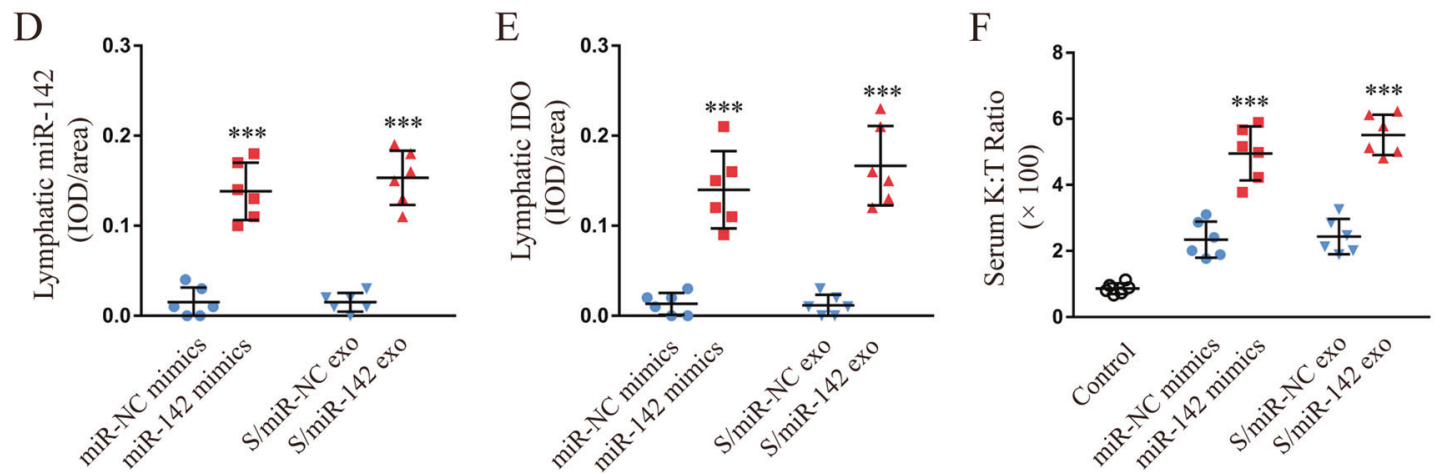

Fig. 3 CSCC-secreted exosomal miR-142-5p up-regulates lymphatic IDO expression. a Protein level of IDO was detected in HDLECs pre-treated with miR-142-5p mimics or indicated exosomes by western blotting and compared with that in the control group. $\mathbf{b}$ The ratio of kynurenine to tryptophan (K:T) in the supernatant of HDLECs $\left(1 \times 10^{5}\right.$ cells $)$ after indicated treatment was calculated. c Representative micrographs of miR-142-5p, IDO, and D240 immunofluorescence staining in sections of xenograft tumours are shown. Scale bar: $50 \mu \mathrm{m}$. d-f The lymphatic expression of miR-142-5 and IDO and serum kynurenine to tryptophan (K:T) ratio in each group were calculated. S/miR-NC Siha/miR-NC, S/miR-142 Siha/miR-142-5p, C/miR-NC Caski/miR-NC, C/miR-142 Caski/miR-142-5p, exo exosomes. Error bars represent the mean $\pm \mathrm{SD}$ of three independent experiments. $* * * P<0.001$.

As a subunit of the polybromo-associated BAF (PBAF) complex, ARID2 potentially interacts with epigenetic regulators and modulates gene transcription [28]. A recent study reported that DNMT1 was recruited as an epigenetic modulator by ARID2 to enhance the methylation of Snail promoter [29]. This observation promoted us to investigate the involvement of the ARID2-DNMT1 complex in the regulation of IFN- $\gamma$ transcription. ChIP-RE-CHIP assay result suggests that DNMT1 and ARID2 co-occupied the 
Fig. 4 CSCC-secreted exosomal miR-142-5p directly targets ARID2 in HDLECs. a Overlap of three miRNA target bioinformatic prediction algorithms. The expression of CCNT2, RAP1A, ARID2, ITGAV, FIGN, and FAM199X in HDLECs pre-treated with miR142-5p mimics or NC was detected by RT-qPCR. b The protein level of ARID2 in HDLECs pre-treated with miR142-5p mimics or NC was analysed by western blotting. c The wild-type- and mutatedbinding sites between miR-142$5 \mathrm{p}$ and ARID2 were shown. d Relative luciferase activity of HDLECs pre-treated with miR142-5p mimics or NC was analysed. e The protein level of ARID2 in HDLECs pre-treated with indicated exosomes was analysed by western blotting. f Relative luciferase activity of HDLECs pre-treated with indicated exosomes was analysed. $\mathbf{g}$ The expression of ARID2 and IDO proteins in miR-142-5p-mimic/exosometreated HDLECs transfected with plasmid-ARID2 or vector control and SiARID2- or SiRNA-treated HDLECs was detected by western blotting. h The K:T ratio of HDLEC supernatant after the indicated treatment was calculated. i IFN$\gamma$ protein level in the supernatant of HDLECs following indicated treatment was detected by ELISA. miR-142, miR-142-5p. S/miR-142 Siha/miR-142-5p, C/miR-NC Caski/miR-NC, C/ miR-142 Caski/miR-142-5p, exo exosomes, WT wild-type, MT mutant. Error bars represent the mean $\pm \mathrm{SD}$ of three independent experiments. $* P<$ $0.05 ; * * P<0.01 ; * * * P<0.001$.
A
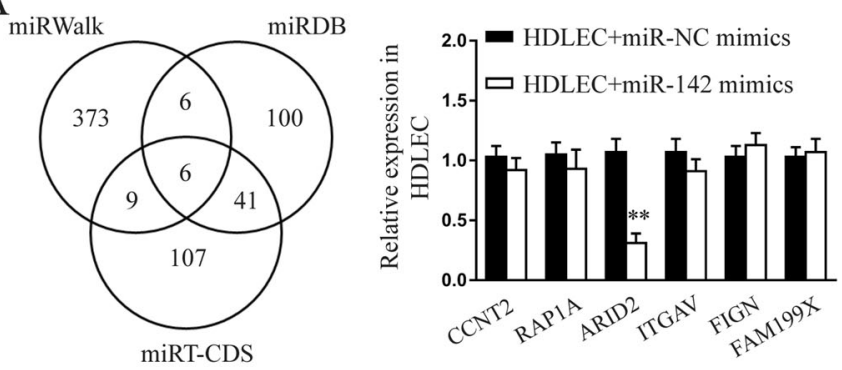

B

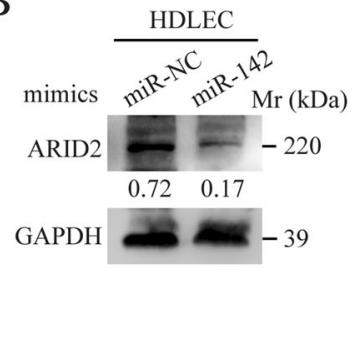

$\mathrm{C}_{\mathrm{A}}$

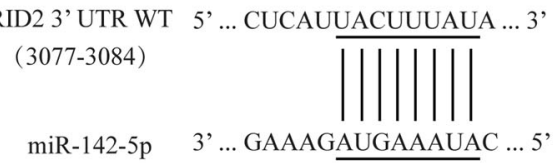

ARID2 3' UTR MT 3' ... GAAAGAUGAAAUAC ... 5',

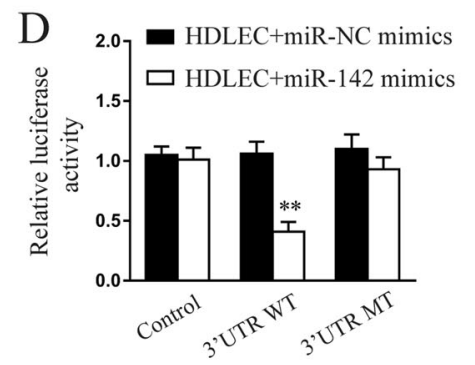

$\mathrm{E}$
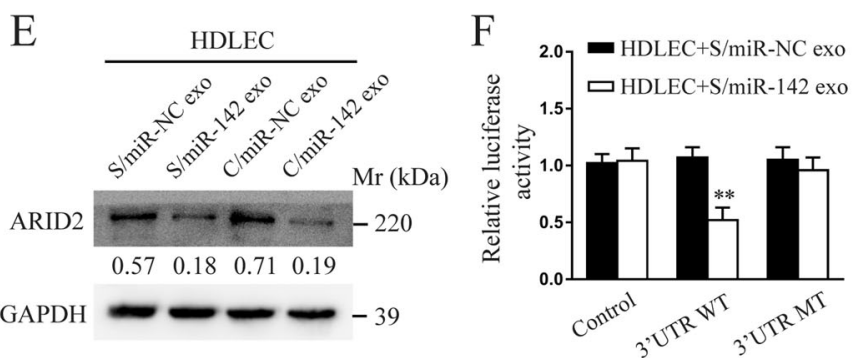

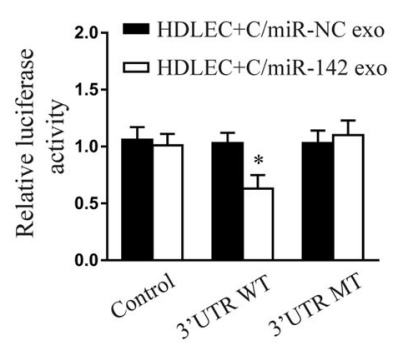

G
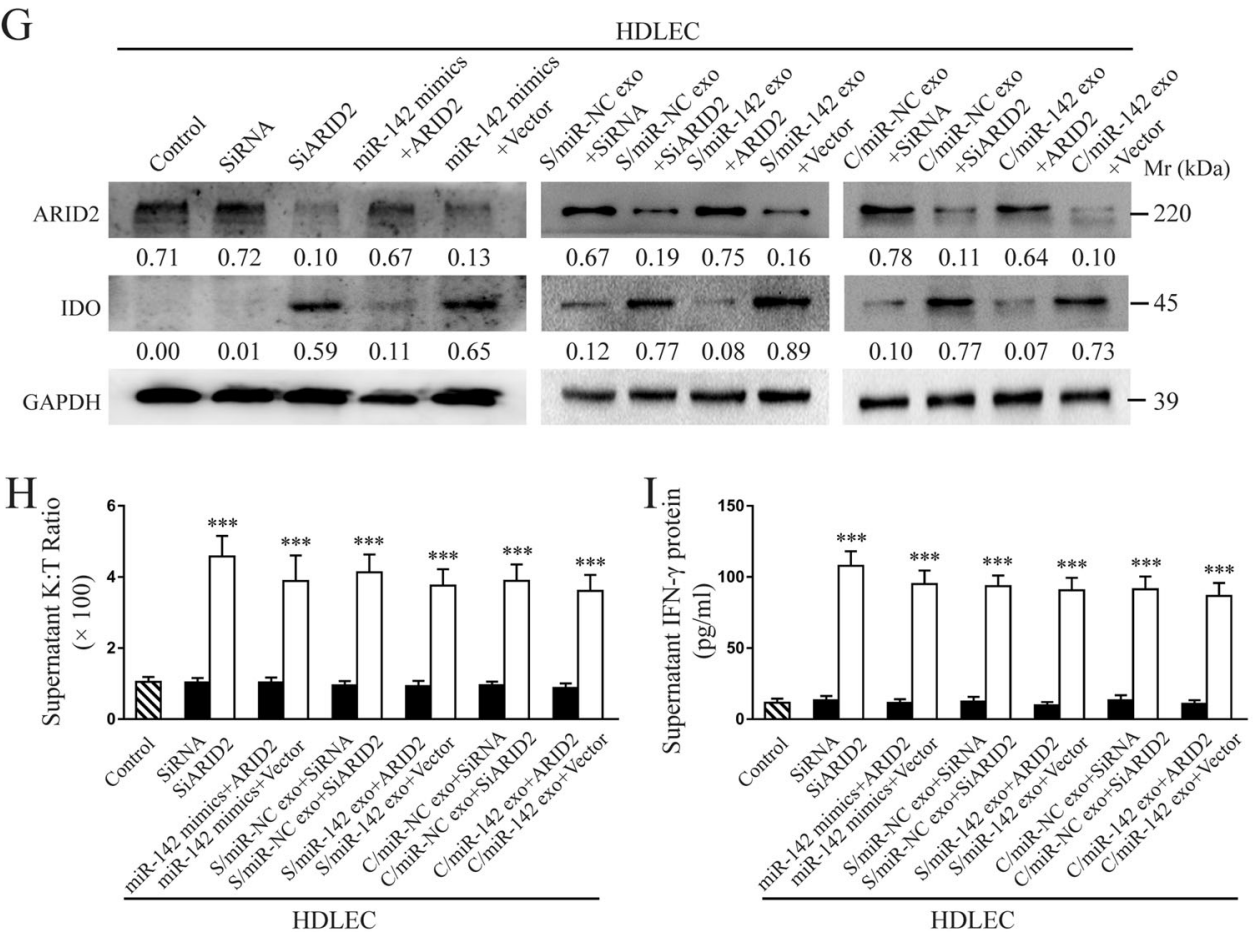

same promoter region of IFN- $\gamma$ (Fig. 5c). Further, the binding of DNMT1 to the IFN- $\gamma$ promoter increased in ARID2-overexpressing HDLECs and decreased in
ARID2-silenced cells, suggesting that ARID2 recruited DNMT1 to the IFN- $\gamma$ promoter (Fig. 5d). We also found that the $\mathrm{CpG}$ island ( -153 to $-277 \mathrm{bp}$ ) overlapped with the 


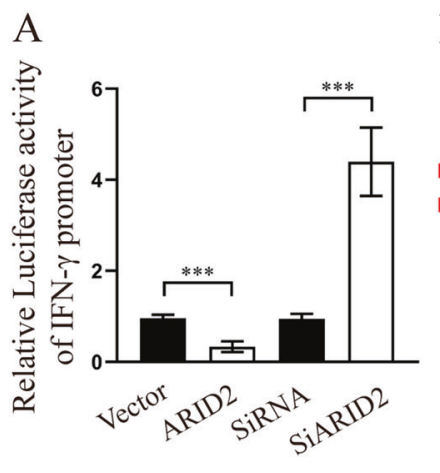

B
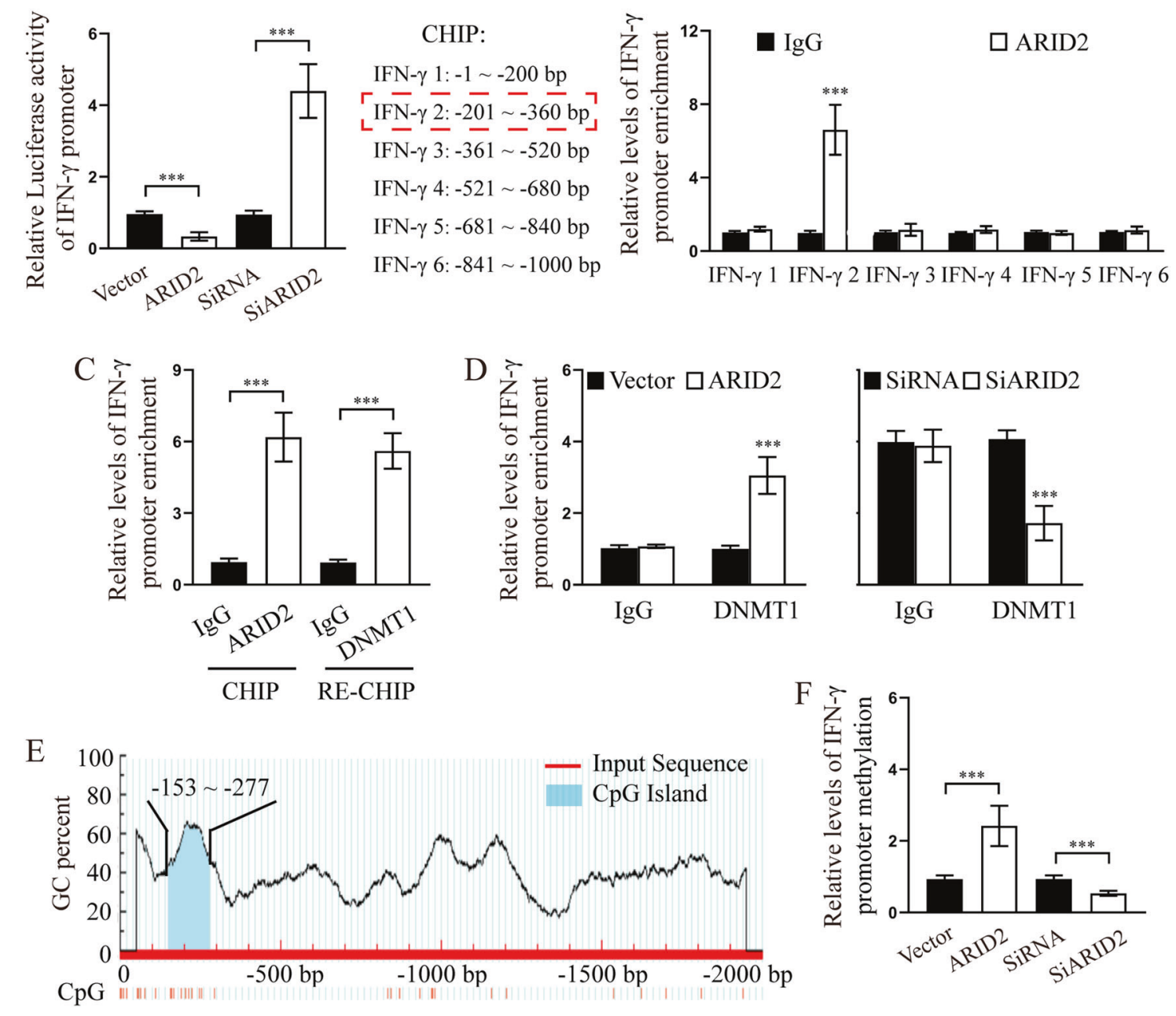

Fig. 5 ARID2 enhances the methylation of IFN- $\gamma$ promoter by recruiting DNMT1. a Relative luciferase activity of IFN- $\gamma$ promoter in HDLECs following ARID2 overexpression or knockdown. b ChIP assay for the enrichment of ARID2 in the promoter region of IFN- $\gamma$ in HDLECs. c ChIP-Re-ChIP assay to determine ARID2-DNMT1 cooccupancy in the promoter region of IFN- $\gamma$. d ChIP assay for the

binding site of the ARID2-DNMT1 complex at the IFN- $\gamma$ promoter ( -201 to $-360 \mathrm{bp}$ ) (Fig. 5e). MSP assay result showed the up-regulation and down-regulation in IFN- $\gamma$ promoter methylation in ARID2-overexpressing and ARID2-silenced HDLECs, respectively, following DNMT1 binding (Fig. 5f). Taken together, our results show that ARID2 suppressed the transcription of IFN- $\gamma$ by recruiting DNMT1 to IFN- $\gamma$ promoter, thereby elevating promoter methylation in HDLECs.

\section{miR-142-5p-activated HDLECs induce IDO expression to exhaust $\mathrm{CDB}^{+} \mathrm{T}$ cells}

IDO promotes immune suppression through the attenuation of $\mathrm{CD}^{+} \mathrm{T}$ cell response [30]. To test whether HDLECs exposed to miR-142-5p contribute to enrichment of DNMT1 in the promoter region of IFN- $\gamma$ in HDLECs following ARID2 overexpression or knockdown. e Schematic map of the $\mathrm{CpG}$ island region in the IFN- $\gamma$ promoter. $\mathrm{f} \mathrm{MSP}$ assay to detect the methylation level of the IFN- $\gamma$ promoter in HDLECs following ARID2 overexpression or knockdown. Error bars represent the mean \pm SD of three independent experiments. $* * * P<0.001$.

suppressive effects on $\mathrm{CD} 8^{+} \mathrm{T}$ cell immunity, HDLECs pre-treated with exosomes with miR-142-5p or miR-142$5 \mathrm{p}$ mimics were co-cultured with in vitro-activated $\mathrm{CD} 8^{+}$ $\mathrm{T}$ cells in the presence of a semipermeable transwell membrane. The $\mathrm{CD} 8^{+} \mathrm{T}$ cells were collected for flow cytometric analysis after culture. HDLECs pre-treated with exosomes containing miR-142-5p showed lower percentages of $\mathrm{CD}^{+} 9^{+}$(activated) and $\mathrm{IFN}-\gamma^{+} \mathrm{CD} 8^{+}$ (effector) T cells compared to the control cells (Fig. 6a, b) and had higher percentages of $\mathrm{PD}-1^{+}$(inhibitory) and annexin $\mathrm{V}^{+}$(apoptotic) $\mathrm{CD}^{+} \mathrm{T}$ cells (Fig. 6c, d). The blockade of IDO or miR-142-5p in HDLECs with an IDO inhibitor (BMS-986205) or miR-142-5p inhibitor (anti142-5p) treatment, respectively, reversed the suppressive effects on $\mathrm{CD}^{+} \mathrm{T}$ cell functions induced by HDLECs pre-treated with exosomes containing high levels of 
A

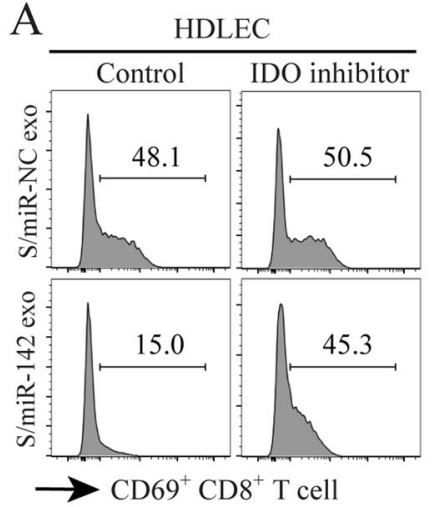

$\mathrm{B}$

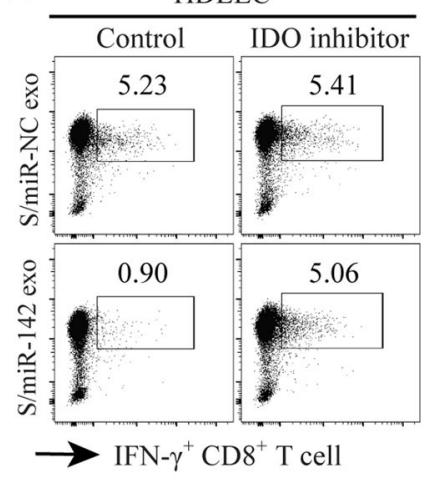

$\mathrm{C}$

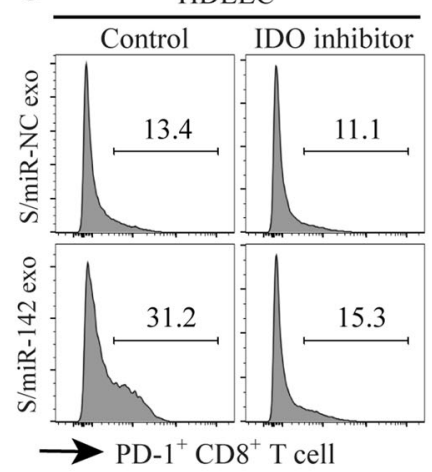

$\mathrm{D}$

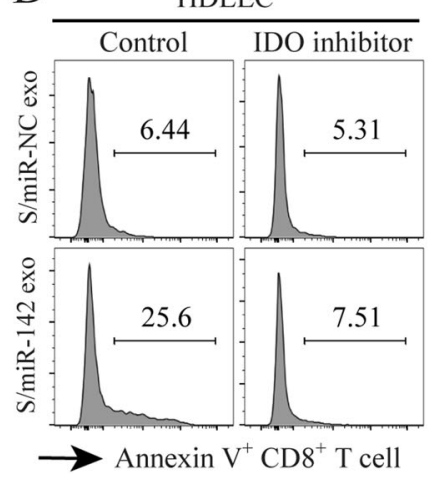

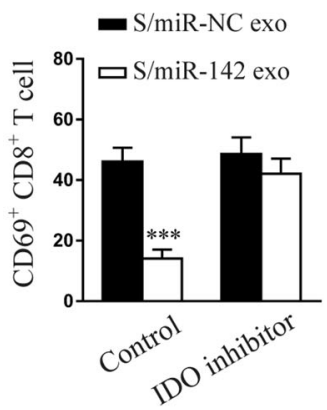
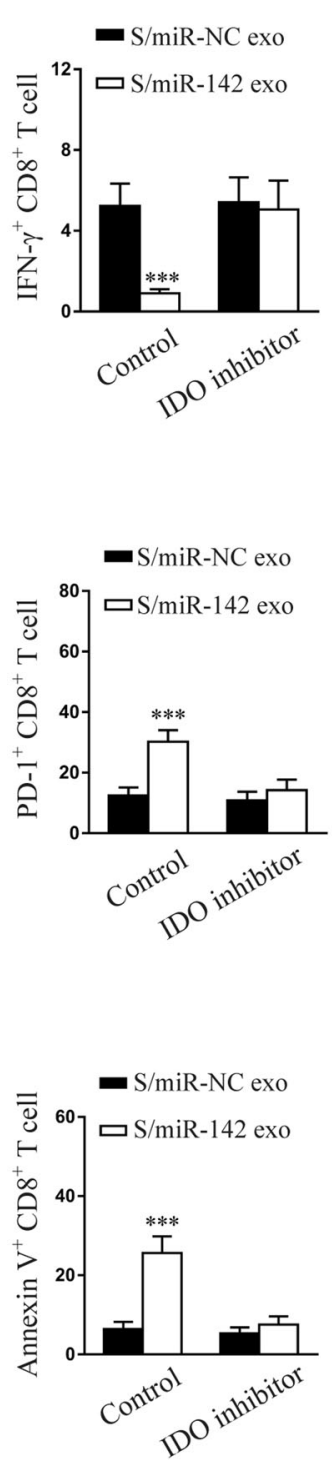

miR-142-5p (Fig. 6a-d; Supplementary Fig. S5a-d). Likewise, HDLECs treated with miR-142-5p mimics also showed a decrease in the percentages of $\mathrm{CD}^{+} 9^{+}$and
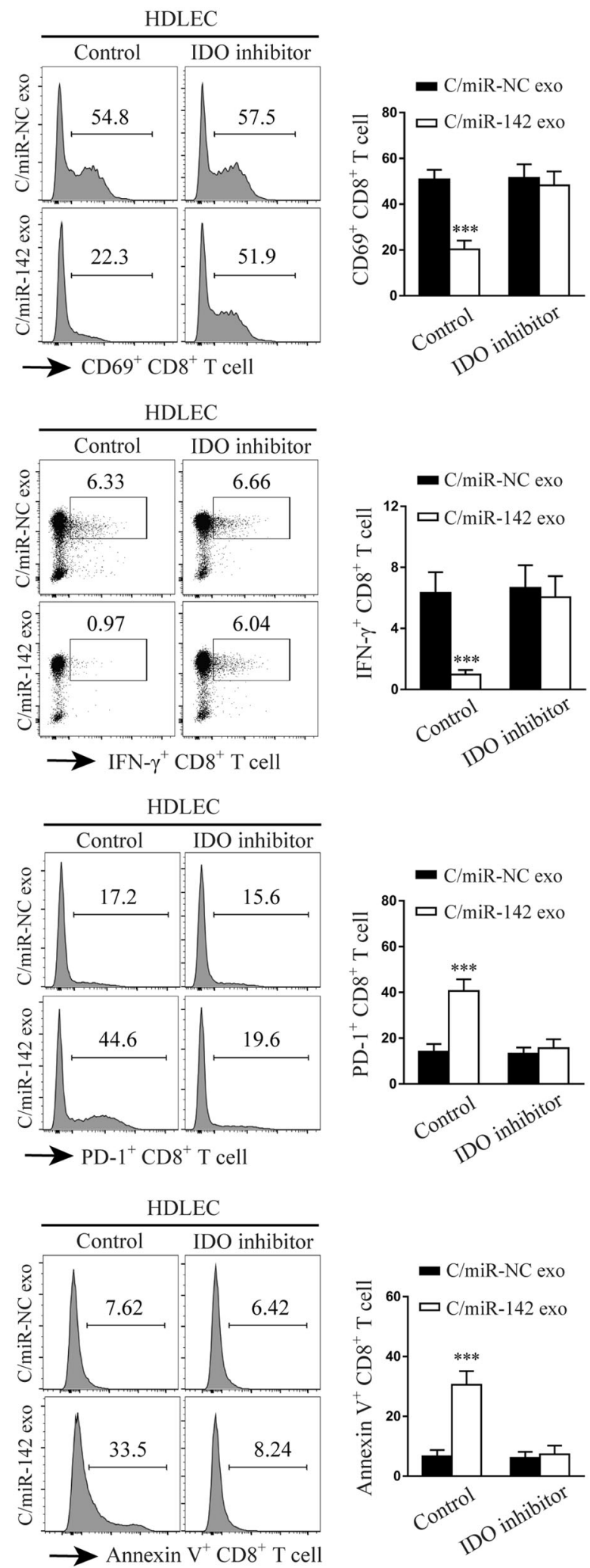

IFN- $\gamma^{+} \mathrm{CD} 8^{+} \mathrm{T}$ cells along with an increase in the percentages of PD- $1^{+}$and annexin $\mathrm{V}^{+} \mathrm{CD}^{+} \mathrm{T}$ cells; these phenomena were reversed after IDO blockade 
Fig. 6 miR-142-5p-activated HDLECs induce IDO to exhaust CD8 $^{+} \mathbf{T}$ cells. HDLECs pre-treated with indicated exosomes were cocultured with in vitro-activated $\mathrm{CD}^{+} \mathrm{T}$ cells at a ratio of $1: 1$ with or without $100 \mathrm{nM}$ of an IDO inhibitor (BMS-986205). The expression of CD69 (a), IFN- $\gamma(\mathbf{b})$, PD-1 (c), and annexin V (d) in CD8 ${ }^{+} \mathrm{T}$ cells was determined by FACS. S/miR-NC exo, Siha/miR-NC exosomes. S/ miR-142 exo, Siha/miR-142-5p exosomes. C/miR-NC exo, Caski/ miR-NC exosomes. C/miR-142 exo, Caski/miR-142-5p exosomes. Error bars represent the mean \pm SD of three independent experiments. $* * * P<0.001$

(Supplementary Fig. S6a-d). Thus, IDO contributes to the immune suppression mediated by miR-142-5p-exposed HDLECs.

\section{Serum exosomal miR-142-5p levels correlate with systemic IDO activity and clinicopathological progression in patients with CSCC}

To investigate whether the cancer-secreted miR-142-5p could be detected in the serum of CSCC patients, the exosomes were isolated from the serum of early-stage (FIGO 2018, stages I and II, $n=27$ ) and advanced-stage (FIGO 2018, stages III and IV, $n=17$ ) CSCC patients as well as healthy controls $(n=8)$ and characterised. The typical morphology and size range of the exosomes purified from the serum were consistent with those of the exosomes derived from cell culture supernatants (Fig. 7a). We then examined exosomal miR-142-5p from these serum samples. As shown in Fig. 7b, the serum exosomal miR-142-5p levels were higher in CSCC patients than in healthy controls. miR-142-5p levels were higher in the serum exosomes from advanced-stage CSCC patients than in those from early-stage CSSC patients. The serum K:T ratio showed a similar trend as serum exosomal miR-142-5p (Fig. 7c). The relationship between exosomal miR-142-5p and K:T ratio in the serum of CSCC patients was further investigated. As shown in Fig. 6d, exosomal miR-142-5p level positively associated with the K:T ratio in the serum of CSCC patients $(r=0.603, P<0.001)$, indicating the potential value of serum exosomal miR-142-5p for the evaluation of IDO activity during CSCC progression.

In summary, our results show that CSCC-secreted exosomal miR-142-5p down-regulates the lymphatic expression of ARID2, inhibits DNMT1 recruitment to the IFN- $\gamma$ promoter, and enhances IFN- $\gamma$ transcription by suppressing promoter methylation, leading to elevated IDO activity to mediate $\mathrm{CD} 8^{+} \mathrm{T}$ cell exhaustion (Fig. 7e).

\section{Discussion}

Multiple studies have demonstrated the clinical relevance of tumour-associated LECs and their involvement in malignant progression [31-33]. While tumour-associated LECs support the migration of tumour cells, they can enhance Foxp3regulatory $\mathrm{T}$ cell functions and suppress responses of effector $\mathrm{T}$ cells, as demonstrated from the gain of a phenotype similar to that of lymphatic node LECs in mice [6, 34]. Our clinical data suggest that tumour-associated LVs helped to modulate anti-tumour immunity and their levels correlated with poor clinical outcome in CSCC patients. Thus, tumour-associated $\mathrm{LVs}$ are an integral component of the immunosuppressive TME. However, the mechanism underlying their function in mediating antitumour immunity is poorly understood.

In a recent study on recurrent or metastatic CSCC, the objective response rate was only $14.3 \%$ after treatment with anti-PD-1 antibodies for the blockade of immune checkpoints despite the selection of patients based on high PD-L1 expression score [35]. These outcomes suggest that additional immune checkpoint(s) mechanisms may control antitumour immunity. Extensive evidence suggests that IDO is associated with immune checkpoint(s) in many cancers [36-38], and our findings reveal the promotion of $\mathrm{CD} 8^{+} \mathrm{T}$ cell exhaustion via IDO mediated by CSCC-remodelled LECs. Although a previous study reported the anti-tumour activity of $\mathrm{IDO}^{+}$LECs in vitro [39], the molecular mechanism leading to lymphatic IDO overexpression in the TME was not identified.

The immune TME is a dynamic system involving a complex intercellular crosstalk that develops and maintains an immune regulatory microenvironment to support tumour growth [40]. Exchange of cellular materials between cells through various paracrine and endocrine mechanisms is an important mean of intercellular crosstalk that may be executed by exosomes [25]. miRNAs from cancer-secreted exosomes are highly versatile regulators of this communication process [41]. In the present study, we found that high miR-142-5p expression correlated with peri-tumoural $\mathrm{IDO}^{+}$LVs during CSCC progression in humans. The role of miR-142-5p as a tumour suppressor or oncogene seems to be cancer type-dependent $[11,12,42]$. Thus, a cancerspecific mechanism underlying the role of miR-142-5p may exist. Our observation that miR-142-5p was directly transferred from tumour cells to LECs via exosomes and upregulated lymphatic IDO expression supported our initial correlative clinical data. In a previous study, we showed that CSCC-secreted exosomal miRNAs served as intercellular regulators between cancer cells and LECs and contributed to the remodelling of peri-tumoural LVs for metastatic spread [43]. Furthermore, high miR-142-5p expression in serum exosomes was associated with elevated IDO activity in advanced cancer stages. A recent study by Ferns et al. supported our idea that IDO activity correlated with poor clinicopathological parameters in CSCC [44]. Our results showed that aside from 

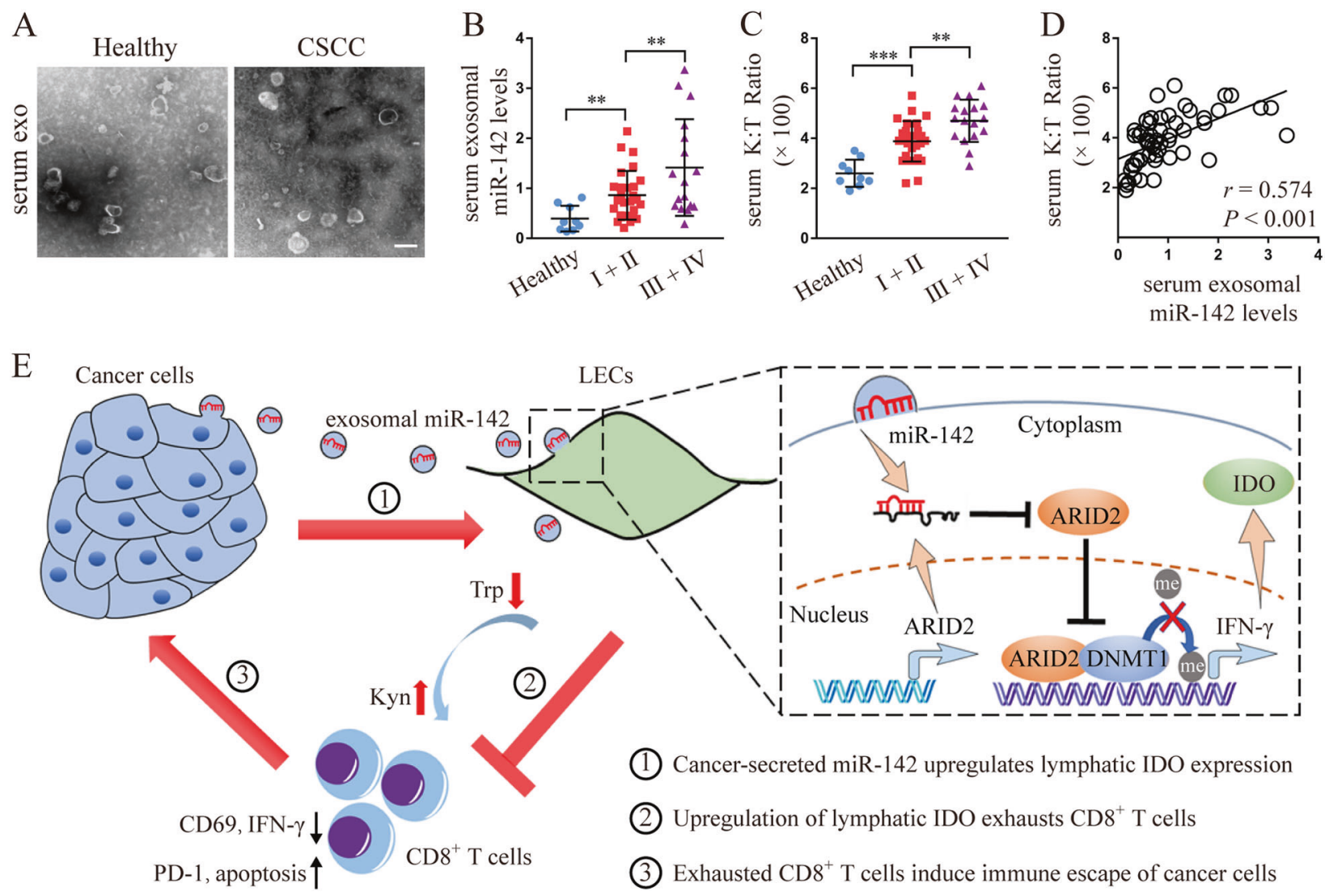

Fig. 7 Serum exosomal miR-142-5p level correlates with systemic IDO activity and clinicopathological progression in patients with CSCC. a Morphology of exosomes isolated and purified from the serum of CSCC patients and healthy controls. Scale Bar: $50 \mathrm{~nm}$. b-c miR-142-5p level and K:T ratio were detected in the serum exosomes of early-stage (FIGO 2018, stages I and II, $n=27$ ) and advanced-stage (FIGO 2018, stages III and IV, $n=17$ ) CSCC patients as well as healthy controls $(n=8)$. d Correlation analysis of exosomal

IDO-mediated anti-tumour immune activity, CSCC malignant progression altered the level of miR-142-5p in serum exosomes, indicating that serum exosomal miR-142-5p may discriminate between indolent and aggressive CSCC and contribute to the development of personalised diagnostic strategies for patients with different progression risks. We found that an IDO inhibitor abrogated the ability of LECs treated with exosomal miR-142-5p to suppress $\mathrm{CD}^{+}$ $\mathrm{T}$ cells. In comparison with an anti-PD-1 treatment alone, the treatment with IDO inhibitor and anti-PD-1 agent increased ORR in CSCC [45]. Thus, therapies targeting miR-142-5p in combination with existing immune therapies such as IDO and PD-1 inhibitors may serve as comprehensive therapeutic approaches for CSCC patients at high risk of progression.

Numerous genes as direct targets of miR-142-5p have been identified. For instance, miR-142-5p directly suppresses the expression of suppressor of cytokine signalling 1 (SOCS1) to control the programming of profibrogenic macrophages via the activation of the signal transducer and activator of transcription (STAT) signalling [46]. Here, we identified a novel target ARID2 as the most down-regulated protein among the several predicted targets associated with miR-142-5p. ARID2 was proven to be a direct target of miR-142-5p by a luciferase reporter assay. In addition, we found that high ARID2 expression correlated with low miR-142-5p levels in human CSCC progression. Members of the ARID family have the ability to regulate transcription and are involved in cell differentiation and proliferation [47]. ARID2 functions as a subunit of the polybromo- and BRG1-associated factor or PBAF (SWI/SNF-B) chromatin remodelling complex, which facilitates ligand-dependent transcriptional activation by nuclear receptors [48]. Several studies have revealed the role of ARID2 as a significant tumour suppressor in many cancer subtypes [49]. Our data suggest that ARID2 protein level can be reduced in LECs by miR$142-5 p$ mimics or exosomes containing miR-142-5p. 
Moreover, overexpression or knockdown of ARID2 could block or replicate the effects induced by miR-142-5p, respectively. IFN- $\gamma$-activated LECs can directly produce IDO in vitro [39], prompting us to determine whether IFN- $\gamma$ could act as a downstream effector of the miR-1425p-ARID2 axis. We found that high levels of miR-142-5p down-regulated ARID2 expression, leading to the upregulation in the expression of IDO mediated by increased IFN- $\gamma$ secretion.

Chromatin remodelling molecules often regulate gene expression by cooperating with epigenetic modulators, such as the E2F4-RBL2-HDAC1-BRM complex involved in proline-rich acidic protein 1 (PRAP1) transcriptional repression [50]. A recent study confirmed the recruitment of DNMT1 as an epigenetic modulator by ARID2 to enhance the methylation of gene promoter [29] that led us to investigate whether the ARID2-DNMT1 complex was also involved in the regulation of IFN- $\gamma$ transcription. We verified the co-occupancy of ARID2 and DNMT1 at the same region of the IFN- $\gamma$ promoter. In addition, the binding of DNMT1 to the IFN- $\gamma$ promoter resulted in an increase in DNA methylation and was regulated by ARID2 expression. These data suggest that DNMT1 was recruited to the IFN- $\gamma$ promoter by ARID2 to enhance promoter methylation.

In conclusion, our data provide evidence for the association of high levels of circulating exosomal miR-142-5p with increased IDO activity in advanced CSCC. The horizontal transfer of CSCC-secreted exosomal miR-142-5p to LECs results in the down-regulation of ARID2 expression, inhibition of DNMT1 recruitment to the IFN- $\gamma$ promoter, and enhancement of IFN- $\gamma$ transcription via the suppression of promoter demethylation, eventually elevating IDO activity for $\mathrm{CD}^{+} \mathrm{T}$ cell exhaustion. The newly identified miR-142-5p-ARID2-DNMT1-IFN- $\gamma$-IDO axis in LECs appears to be a critical molecular mechanism underlying CSCC progression and may serve as a novel diagnostic and therapeutic target for CSCC patients at high risk of progression.

Acknowledgements We thank Professor Andrew L. Mellor for editing the English writing.

Funding This work was supported by the National Natural Science Foundation of China [Grant nos. 81672589, 81971341, and 81902621] and China Postdoctoral Science Foundation [Grant nos. 2019M662868 and 2019M662867].

\section{Compliance with ethical standards}

Conflict of interest ALM receives patent licensing income from NewLink Genetics Inc. All other authors declare no conflict of interest.

Publisher's note Springer Nature remains neutral with regard to jurisdictional claims in published maps and institutional affiliations.
Open Access This article is licensed under a Creative Commons Attribution 4.0 International License, which permits use, sharing, adaptation, distribution and reproduction in any medium or format, as long as you give appropriate credit to the original author(s) and the source, provide a link to the Creative Commons license, and indicate if changes were made. The images or other third party material in this article are included in the article's Creative Commons license, unless indicated otherwise in a credit line to the material. If material is not included in the article's Creative Commons license and your intended use is not permitted by statutory regulation or exceeds the permitted use, you will need to obtain permission directly from the copyright holder. To view a copy of this license, visit http://creativecommons. org/licenses/by/4.0/.

\section{References}

1. Zhou W, Fong MY, Min Y, Somlo G, Liu L, Palomares MR, et al. Cancer-secreted miR-105 destroys vascular endothelial barriers to promote metastasis. Cancer Cell. 2014;25:501-15.

2. Wei JC, Yang J, Liu D, Wu MF, Qiao L, Wang JN, et al. Tumorassociated lymphatic endothelial cells promote lymphatic metastasis by highly expressing and secreting SEMA4C. Clin Cancer Res. 2017;23:214-24.

3. Kato T, Noma K, Ohara T, Kashima H, Katsura Y, Sato H, et al. Cancer-associated fibroblasts affect intratumoral $\mathrm{CD} 8(+)$ and FoxP3(+) T cells via IL6 in the tumor microenvironment. Clin Cancer Res. 2018;24:4820-33.

4. Stacker SA, Williams SP, Karnezis T, Shayan R, Fox SB, Achen MG. Lymphangiogenesis and lymphatic vessel remodelling in cancer. Nat Rev Cancer. 2014;14:159-72.

5. Ma Q, Dieterich LC, Detmar M. Multiple roles of lymphatic vessels in tumor progression. Curr Opin Immunol. 2018;53:7-12.

6. Dieterich LC, Ikenberg K, Cetintas T, Kapaklikaya K, Hutmacher C, Detmar M. Tumor-associated lymphatic vessels upregulate PDL1 to inhibit T-cell activation. Front Immunol. 2017;8:66.

7. Liu Y, Luo F, Wang B, Li H, Xu Y, Liu X, et al. STAT3-regulated exosomal miR-21 promotes angiogenesis and is involved in neoplastic processes of transformed human bronchial epithelial cells. Cancer Lett. 2016;370:125-35.

8. Vickers KC, Palmisano BT, Shoucri BM, Shamburek RD, Remaley AT. MicroRNAs are transported in plasma and delivered to recipient cells by high-density lipoproteins. Nat Cell Biol. 2011;13:423-33.

9. Redis RS, Calin S, Yang Y, You MJ, Calin GA. Cell-to-cell miRNA transfer: from body homeostasis to therapy. Pharm Ther. 2012;136:169-74.

10. Yan J, Yang B, Lin S, Xing R, Lu Y. Downregulation of miR-142$5 p$ promotes tumor metastasis through directly regulating CYR61 expression in gastric cancer. Gastric Cancer. 2019;22:302-13.

11. Liu L, Liu S, Duan Q, Chen L, Wu T, Qian H, et al. MicroRNA$142-5 p$ promotes cell growth and migration in renal cell carcinoma by targeting BTG3. Am J Transl Res. 2017;9:2394-402.

12. Liu S, Xiao Z, Ai F, Liu F, Chen X, Cao K, et al. miR-142-5p promotes development of colorectal cancer through targeting SDHB and facilitating generation of aerobic glycolysis. Biomed Pharmacother. 2017;92:1119-27.

13. Bai X, Zhou Y, Chen P, Yang M, Xu J. MicroRNA-142-5p induces cancer stem cell-like properties of cutaneous squamous cell carcinoma via inhibiting PTEN. J Cell Biochem. 2018;119:2179-88.

14. Platten M, Wick W, Van den Eynde BJ. Tryptophan catabolism in cancer: beyond IDO and tryptophan depletion. Cancer Res. 2012;72:5435-40.

15. Wang W, Huang L, Jin JY, Jolly S, Zang Y, Wu H, et al. IDO immune status after chemoradiation may predict survival in lung cancer patients. Cancer Res. 2018;78:809-16. 
16. Zhang G, Liu WL, Zhang L, Wang JY, Kuang MH, Liu P, et al. Involvement of indoleamine 2,3-dioxygenase in impairing tumorinfiltrating CD8 T-cell functions in esophageal squamous cell carcinoma. Clin Dev Immunol. 2011;2011:384726.

17. Ino K, Yamamoto E, Shibata K, Kajiyama H, Yoshida N, Terauchi $\mathrm{M}$, et al. Inverse correlation between tumoral indoleamine 2,3-dioxygenase expression and tumor-infiltrating lymphocytes in endometrial cancer: its association with disease progression and survival. Clin Cancer Res. 2008;14:2310-7.

18. Uyttenhove C, Pilotte L, Theate I, Stroobant V, Colau D, Parmentier $\mathrm{N}$, et al. Evidence for a tumoral immune resistance mechanism based on tryptophan degradation by indoleamine 2,3dioxygenase. Nat Med. 2003;9:1269-74.

19. Riesenberg R, Weiler C, Spring O, Eder M, Buchner A, Popp T, et al. Expression of indoleamine 2,3-dioxygenase in tumor endothelial cells correlates with long-term survival of patients with renal cell carcinoma. Clin Cancer Res. 2007;13:6993-7002.

20. Pelak MJ, Snietura M, Lange D, Nikiel B, Pecka KM. The prognostic significance of indoleamine-2,3-dioxygenase and the receptors for transforming growth factor beta and interferon gamma in metastatic lymph nodes in malignant melanoma. Pol $\mathbf{J}$ Pathol. 2015;66:376-82.

21. Dill EA, Dillon PM, Bullock TN, Mills AM. IDO expression in breast cancer: an assessment of 281 primary and metastatic cases with comparison to PD-L1. Mod Pathol. 2018;31:1513-22.

22. Chinnadurai R, Rajan D, Ng S, McCullough K, Arafat D, Waller $\mathrm{EK}$, et al. Immune dysfunctionality of replicative senescent mesenchymal stromal cells is corrected by IFNgamma priming. Blood Adv. 2017;1:628-43.

23. Remmele W, Schicketanz KH. Immunohistochemical determination of estrogen and progesterone receptor content in human breast cancer. Computer-assisted image analysis (QIC score) vs. subjective grading (IRS). Pathol Res Pr. 1993;189:862-6.

24. Roh MR, Kumar R, Rajadurai A, Njauw C, Ryoo UH, Chung KY, et al. Beta-catenin causes fibrotic changes in the extracellular matrix via upregulation of collagen I transcription. Br J Dermatol. 2017;177:312-5.

25. Naito Y, Yoshioka Y, Yamamoto Y, Ochiya T. How cancer cells dictate their microenvironment: present roles of extracellular vesicles. Cell Mol Life Sci. 2017;74:697-713.

26. Ricciuti B, Leonardi GC, Puccetti P, Fallarino F, Bianconi V, Sahebkar A, et al. Targeting indoleamine-2,3-dioxygenase in cancer: Scientific rationale and clinical evidence. Pharm Ther. 2018;196:105-16.

27. Lu J, Liu X, Liao YP, Wang X, Ahmed A, Jiang W, et al. Breast cancer chemo-immunotherapy through liposomal delivery of an immunogenic cell death stimulus plus interference in the IDO-1 pathway. ACS Nano. 2018;12:11041-61.

28. Wilson BG, Roberts CW. SWI/SNF nucleosome remodellers and cancer. Nat Rev Cancer. 2011;11:481-92.

29. Jiang H, Cao HJ, Ma N, Bao WD, Wang JJ, Chen TW, et al. Chromatin remodeling factor ARID2 suppresses hepatocellular carcinoma metastasis via DNMT1-Snail axis. Proc Natl Acad Sci USA. 2020;117:4770-80.

30. Munn DH, Mellor AL. IDO in the tumor microenvironment: inflammation, counter-regulation, and tolerance. Trends Immunol. 2016;37:193-207.

31. Liu D, Li L, Zhang XX, Wan DY, Xi BX, Hu Z, et al. SIX1 promotes tumor lymphangiogenesis by coordinating TGFbeta signals that increase expression of VEGF-C. Cancer Res. 2014;74:5597-607.

32. Liu L, Lin C, Liang W, Wu S, Liu A, Wu J, et al. TBL1XR1 promotes lymphangiogenesis and lymphatic metastasis in esophageal squamous cell carcinoma. Gut. 2015;64:26-36.
33. Wang SH, Chang JS, Hsiao JR, Yen YC, Jiang SS, Liu SH, et al. Tumour cell-derived WNT5B modulates in vitro lymphangiogenesis via induction of partial endothelial-mesenchymal transition of lymphatic endothelial cells. Oncogene. 2017;36:1503-15.

34. Lund AW, Duraes FV, Hirosue S, Raghavan VR, Nembrini C, Thomas SN, et al. VEGF-C promotes immune tolerance in B16 melanomas and cross-presentation of tumor antigen by lymph node lymphatics. Cell Rep. 2012;1:191-9.

35. Theodorescu D. Pembrolizumab OK'd for cervical cancer. Cancer Disco. 2018;8:904.

36. Spranger S, Spaapen RM, Zha Y, Williams J, Meng Y, Ha TT, et al. Up-regulation of PD-L1, IDO, and T(regs) in the melanoma tumor microenvironment is driven by CD8(+) T cells. Sci Transl Med. 2013;5:200ra116.

37. Han Y, Chen Z, Yang Y, Jiang Z, Gu Y, Liu Y, et al. Human CD14+ CTLA-4+ regulatory dendritic cells suppress T-cell response by cytotoxic T-lymphocyte antigen-4-dependent IL-10 and indoleamine-2,3-dioxygenase production in hepatocellular carcinoma. Hepatology. 2014;59:567-79.

38. Triplett TA, Garrison KC, Marshall N, Donkor M, Blazeck J, Lamb C, et al. Reversal of indoleamine 2,3-dioxygenase-mediated cancer immune suppression by systemic kynurenine depletion with a therapeutic enzyme. Nat Biotechnol. 2018;36:758-64.

39. Norder M, Gutierrez MG, Zicari S, Cervi E, Caruso A, Guzman CA. Lymph node-derived lymphatic endothelial cells express functional costimulatory molecules and impair dendritic cellinduced allogenic T-cell proliferation. Faseb J. 2012;26:2835-46.

40. Binnewies M, Roberts EW, Kersten K, Chan V, Fearon DF, Merad $M$, et al. Understanding the tumor immune microenvironment (TIME) for effective therapy. Nat Med. 2018;24:541-50.

41. Takahashi RU, Prieto-Vila M, Hironaka A, Ochiya T. The role of extracellular vesicle microRNAs in cancer biology. Clin Chem Lab Med. 2017;55:648-56.

42. Yan J, Yang B, Lin S, Xing R, Lu Y. Downregulation of miR$142-5 p$ promotes tumor metastasis through directly regulating CYR61 expression in gastric cancer. Gastric Cancer. 2018;22:302-13.

43. Zhou CF, Ma J, Huang L, Yi HY, Zhang YM, Wu XG, et al. Cervical squamous cell carcinoma-secreted exosomal miR-221-3p promotes lymphangiogenesis and lymphatic metastasis by targeting VASH1. Oncogene. 2019;38:1256-68.

44. Ferns DM, Kema IP, Buist MR, Nijman HW, Kenter GG, Jordanova ES. Indoleamine-2,3-dioxygenase (IDO) metabolic activity is detrimental for cervical cancer patient survival. Oncoimmunology. 2015;4:e981457.

45. Blocking IDO1 helps shrink bladder, Cervical Tumors. Cancer Discov. 2018;8:Of3.

46. Su S, Zhao Q, He C, Huang D, Liu J, Chen F, et al. miR-142-5p and miR-130a-3p are regulated by IL- 4 and IL-13 and control profibrogenic macrophage program. Nat Commun. 2015;6:8523.

47. Lin C, Song W, Bi X, Zhao J, Huang Z, Li Z, et al. Recent advances in the ARID family: focusing on roles in human cancer. Onco Targets Ther. 2014;7:315-24.

48. Hodges C, Kirkland JG, Crabtree GR. The many roles of BAF (mSWI/SNF) and PBAF complexes in cancer. Cold Spring Harb Perspect Med. 2016;6:a026930.

49. Hodis E, Watson IR, Kryukov GV, Arold ST, Imielinski M, Theurillat JP, et al. A landscape of driver mutations in melanoma. Cell. 2012;150:251-63.

50. Wiśnik E, Płoszaj T, Robaszkiewicz A. Downregulation of PARP1 transcription by promoter-associated E2F4-RBL2HDAC1-BRM complex contributes to repression of pluripotency stem cell factors in human monocytes. Sci Rep. 2017;7:9483. 